\title{
Ligadura do canal arterial: técnica extrapleural
}

José Luiz Verde dos SANTOS*, Domingo M. BRAILE*, Roberto V. ARDITO* Marcos ZAIANTCHICK*, Marcelo José F. SOARES*, Walter RADE*, João Carlos Ferreira LEAL*, José Luiz B. JACOB*, Sírio HASSEN SOBRINHO*

RBCCV 44205-157

SANTOS, J.L.V.; BRAILE, D.M.; ARDITO, R.V.; ZAIANTCHICK, M.; SOARES, M.J.F.; RADE, W.; LEAL, J.C.F.; JACOB, J.L.B.; HASSEN SOBRINHO, S. - Ligadura do canal arterial: técnica extrapleural. Rev. Bras. Cir. Cardiovasc., 7(1):14-21, 1992.

RESUMO: A técnica de correção cirúrgica da persistência do canal arterial (PCA) com ligadura tripla por via extrapleural é apresentada. Após anestesia geral, a criança é posicionada em decúbito lateral direito tendendo a decúbito ventral. Por uma pequena incisão acompanhando a borda inferior da escápula, o canal arterial é dissecado e ligado duplamente com fios Poliester $2 / 0$ e um ponto transfixante de Polipropileno 4/0. Essa técnica dispensa drenagem torácica. De setembro de 1988 a agosto de 1990, foram operados 47 pacientes portadores de PCA. Destes, 40 foram submetidos à técnica. A idade variou de quatro meses a 11 anos com média de 3,1 $\pm 3,0$ anos (Tabela 1). Vinte e quatro crianças eram do sexo feminino e 16 do masculino. Vinte crianças $(50 \%)$ tinham idade menor que um ano. Não foi registrada nenhuma intercorrência intra ou pósoperatória. Todas as crianças foram seguidas no pós-operatório tardio com avaliaçảo clínica, exame radiológico do tórax e ecodopplercardiografia. Nenhuma delas apresentou recanalização ou lesão de nervo recorrente, num seguimento que variou de sete a 30 meses, com média de 15,3 meses. Em nossa experiência, a ligadura do canal arterial mostrou-se benigna quanto ao intra e ao pós-operatório, não apresentando mortalidade imediata ou tardia. Essa técnica deve ser utilizada como de escolha na correção da PCA, principalmente com crianças de baixa idade e peso.

DESCRITORES: persistência de canal arterial, cirurgia.

\section{INTRODUÇĀO}

O canal arterial corre paralelamente ao arco aórtico, desde a artéria pulmonar esquerda, passa pelo pericárdio e une-se à margem medial da aorta em ângulo agudo, opondo-se à origem da artéria esquerda ${ }^{9}$.

Sua histologia difere da aorta e da artéria pulmonar por apresentar íntima espessada com uma única camada elástica na média, além de conter substância mucóide e arranjo muscular helicoidal.

Seu fechamento espontâneo ocorre imediatamente após o nascimento, por contração muscular de sua parede ${ }^{12}$. Nos primeiros dias de vida, não é incomum um certo fluxo aortopulmonar. O tempo de obliteração por proliferação da íntima ocorre em um a três meses, transformando o canal em ligamento arterioso.

A ausência de fechamento espontâneo do canal arterial leva a graus variáveis de hipervolemia e hiperfluxo pulmonar. Essa persistência é responsável por $12,0 \%$ das cardiopatias congênitas, com preferência pelo sexo feminino na proporção de 2:1. Apenas $2,0 \%$ dos casos estão relacionados a rubéola congênita ${ }^{12}$.

Várias técnicas têm sido propostas, desde 1939,

Trabalho realizado no Instituto de Moléstias Cardiovasculares. São José do Rio Preto, SP, Brasil.

Recebido para publicação em 30 de janeiro de 1992.

* Do Instituto de Molésticas Cardiovasculares.

Endereço para separatas: José Luiz Verde dos Santos. Instituto do Coração de Presidente Prudente. Rua Donato Armelin, 351.19013 Presidente Prudente, SP, Brasil. 
SANTOS, J.L.V.; BRAILE, D.M.; ARDITO, R.V.; ZAIANTCHICK, M.; SOARES, M.J.F.; RADE, W.; LEAL, J.C.F.; JACOB, J.L.B.; HASSEN SOBRINHO, S. - Ligadura do canal arterial: técnica extrapleural. Rev. Bras. Cir. Cardiovasc., 7(1):14-21, 1992.

TABELA 1

CRIANCAS SUBMETIDAS A LIGADURA DO CANAL ARTERIAL PELA TÉCNICA EXTRAPLEURAL E SEU SEGUIMENTO TARDIO

\begin{tabular}{|c|c|c|}
\hline NOME & $I D A D E$ & SEGUIMENTO (MESES) \\
\hline P.C.P. & $10 a$ & 30 \\
\hline F.S.L. & $6 a$ & 29 \\
\hline T.S.D.\#** & $1 a$ & 29 \\
\hline G.R. & $6 a$ & 29 \\
\hline S.C.B. & $1 a$ & 28 \\
\hline C.C.G. & $4 \mathrm{~m}$ & 26 \\
\hline E.F.N. & $10 \mathrm{~m}$ & 26 \\
\hline D.A. & $1 \mathbf{a}$ & 25 \\
\hline A.S.G. & 8 a & 25 \\
\hline J.C.C.S. & $1 a$ & 25 \\
\hline 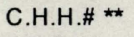 & $6 a$ & 25 \\
\hline J.R.R. *^ & $4 a$ & 24 \\
\hline A.D.D. ${ }^{\star \star}$ & $10 \mathrm{a}$ & 23 \\
\hline W.R.M. & $10 \mathrm{~m}$ & 23 \\
\hline S.V.C. ${ }^{\star \star}$ & $7 \mathbf{a}$ & 20 \\
\hline L.C.P. & $8 a$ & 19 \\
\hline M.A.P. & $2 a$ & 19 \\
\hline M.I.P. & $2 a$ & 19 \\
\hline M.C.O. ** & $4 a$ & 18 \\
\hline D.B.C. & $1 a$ & 18 \\
\hline R.D.P.\# ${ }^{\star \star}$ & $1 a$ & 17 \\
\hline V.R.F.R. & 6 a & 17 \\
\hline D.S.S.\# ** & $2 a$ & 17 \\
\hline M.S.G. ** & $1 a$ & 17 \\
\hline F.C.A. & $2 a$ & 16 \\
\hline P.H.S. & $1 a$ & 16 \\
\hline D.O.F. ** & $9 \mathrm{~m}$ & 16 \\
\hline A.A.R. & $1 a$ & 16 \\
\hline R.K. & $1 a$ & 16 \\
\hline S.F.S. ${ }^{\star \star}$ & $1 a$ & 15 \\
\hline R.A.M. & $2 a$ & 13 \\
\hline W.V.M.** & $6 \mathrm{~m}$ & 13 \\
\hline J.V.S. & $3 a$ & 12 \\
\hline J.O.S. & $4 a$ & 12 \\
\hline R.A.S. & $1 a$ & 12 \\
\hline P.A.R. ${ }^{\star \star}$ & $2 a$ & 11 \\
\hline R.R.A. & $7 \mathrm{~m}$ & 9 \\
\hline A.I.G. ${ }^{\star \star}$ & $1 a$ & 8 \\
\hline L.S.O.** & $1 a$ & 7 \\
\hline
\end{tabular}

\# Diagnóstico confirmado por estudo hemodinâmico

** Presença de lesão associada.

quando GROSS \& HUBBARD ${ }^{8}$ registraram a primeira cirurgia de ligadura do canal arterial com sucesso, entre elas: ligadura dupla, ligadura tripla, uso de clips metálicos e secção e sutura.

Apresentamos uma técnica de ligadura tripla por abordagem extrapleural. Esse tipo de abordagem cirúrgica vem sendo realizado, em nosso Serviço, desde 1988, sem complicações, e é simples de ser realizada.

\section{CASUÍSTICA E MÉTODOS}

De setembro de 1988 a agosto de 1990, foram submetidas a correção cirúrgica do canal arterial 46 crianças e um adulto, sendo $40 \mathrm{com}$ a técnica extrapleural. A idade variou de quatro meses a 11 anos, com média de $3,1 \pm 3,0$ anos, sendo 16 do sexo masculino e 24 do feminino. Vinte crianças $(50,0 \%)$ tinham idade menor ou igual a um ano.

O diagnóstico de persistência do canal arterial foi sempre realizado por história clínica e ausculta de sopro contínuo em maquinária no foco pulmonar com irradiação para o dorso. Foi complementado pelo exame radiológico do tórax (Figura 1), que mostrava sinais de hiperfluxo pulmonar de grau variável, confirmado pelo ecodopplercardiograma bidimensional, que evidenciava fluxo contínuo aortopulmonar. Apenas quatro crianças foram submetidas a estudo hemodinâmico, por serem portadoras de outras cardiopatias congênitas associadas. A Tabela 2 mostra o número e tipo de associaçōes ao canal arterial.

A indicação de ligadura do canal arterial utilizando a técnica aqui descrita foi feita de acordo com quadro clínico, exame físico, radiografia de tórax, ecodopplecardiografia e estudo hemodinâmico, quando houve suspeita de cardiopatia congênita associada.

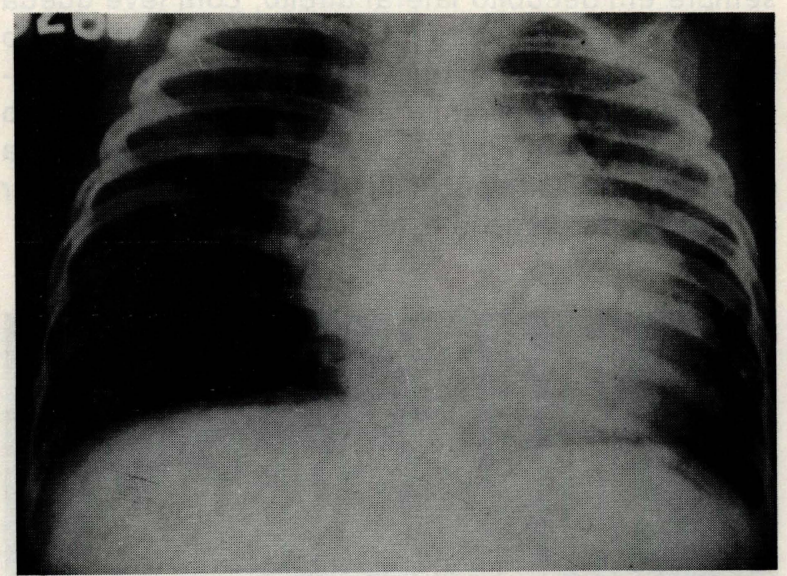

Fig. 1 - Radiografia torácica póstero-anterior de criança de dois anos de idade portadora de persistência do canal arterial, mostrando congestão pulmonar por hipervolemia (hiperfluxo) e cardiomegalia.

TABELA 2

CRIANÇAS PORTADORAS DE CARDIOPATIAS CONGÊNITAS ASSOCIADAS A PERSISTENNCIA DO CANAL ARTERIAL SUBMETIDAS À TÉCNICA DE LIGADURA EXTRAPLEURAL

\begin{tabular}{lc}
\hline PATOLOGIA ASSOCIADA & No CRIANÇAS \\
\hline Estenose Pulmonar & 6 \\
Rubéola Congênita & 3 \\
Valva Aórtica Bicúspide & 3 \\
Estenose Sub-aórtica & 1 \\
Síndrome de Marfan & 1 \\
Insuficiência Aórtica & 1 \\
Comunicação Interventricular & 1 \\
Insuficiência Mitral Discreta & 1 \\
TOTAL & $17 \quad(43,6 \%)$ \\
\hline
\end{tabular}


SANTOS, J.L.V.; BRAILE, D.M.; ARDITO, R.V.; ZAIANTCHICK, M.; SOARES, M.J.F.; RADE, W.; LEAL, J.C.F.; JACOB, J.L.B.; HASSEN SOBRINHO, S. - Ligadura do canal arterial: técnica extrapleural. Rev. Bras. Cir. Cardiovasc., 7(1):14-21, 1992.

\section{Monitoração e Indução Anestésica}

As crianças foram trazidas ao centro cirúrgico nos braços da mãe, quando foi o caso, onde receberam medicação pré-anestésica (Ketamina $7 \mathrm{mg} / \mathrm{kg}$ ) intramuscular. Aguardado o efeito, os pacientes foram levados para a mesa cirúrgica e submetidos a monitorização eletrocardiográfica e indução anestésica com Halotano sob máscara, permitindo a dissecção venosa. Foi utilizado, para controle da diurese, preferencialmente coletor urinário, evitando conduta invasiva desnecessária, diante do curto tempo cirúrgico ( 30 a 40 minutos). Não se realizou, necessariamente, dissecção ou punção arterial. Os pacientes foram posicionados para a intervenção após entubação orotraqueal e passagem de sonda nasogástrica. Utilizou-se termômetro retal para monitorizar a temperatura corporal, controlada por colchão térmico.

\section{Técnica Cirúrgica}

A posição do paciente na mesa cirúrgica foi sempre em decúbito lateral direito, com leve queda para frente (tendência a decúbito ventral). $O$ membro superior esquerdo foi tracionado para cima da cabeça, para que a borda inferior da escápula marcasse o local da incisão (Figura 2). A antissepsia foi realizada com tintura de iodo polivinilpirrolidona, com posterior colocação de campos estéreis.

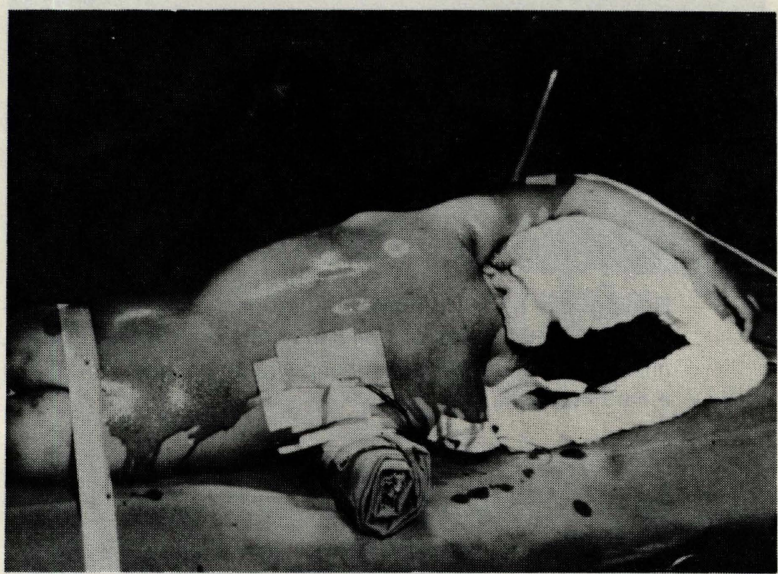

Fig. 2 - Posição da criança na mesa cirúrgica: decúbito lateral direito tendendo a decúbito dorsal; tração do membro superior esquerdo para cima da cabeça para que a borda inferior da escápula marque o local da incisão.

A incisão foi feita acompanhando a borda inferior da escápula. O tamanho da incisão, pouco menor que a borda, depende do tamanho da criança, variando de 2,5 a $5 \mathrm{~cm}$ (Figura 3 ). Os músculos grande dorsal e redondo maior foram incisados ou afastados, dando acesso ao gradil costal. A abertura da cavidade torácica foi realizada através do terceiro espaço

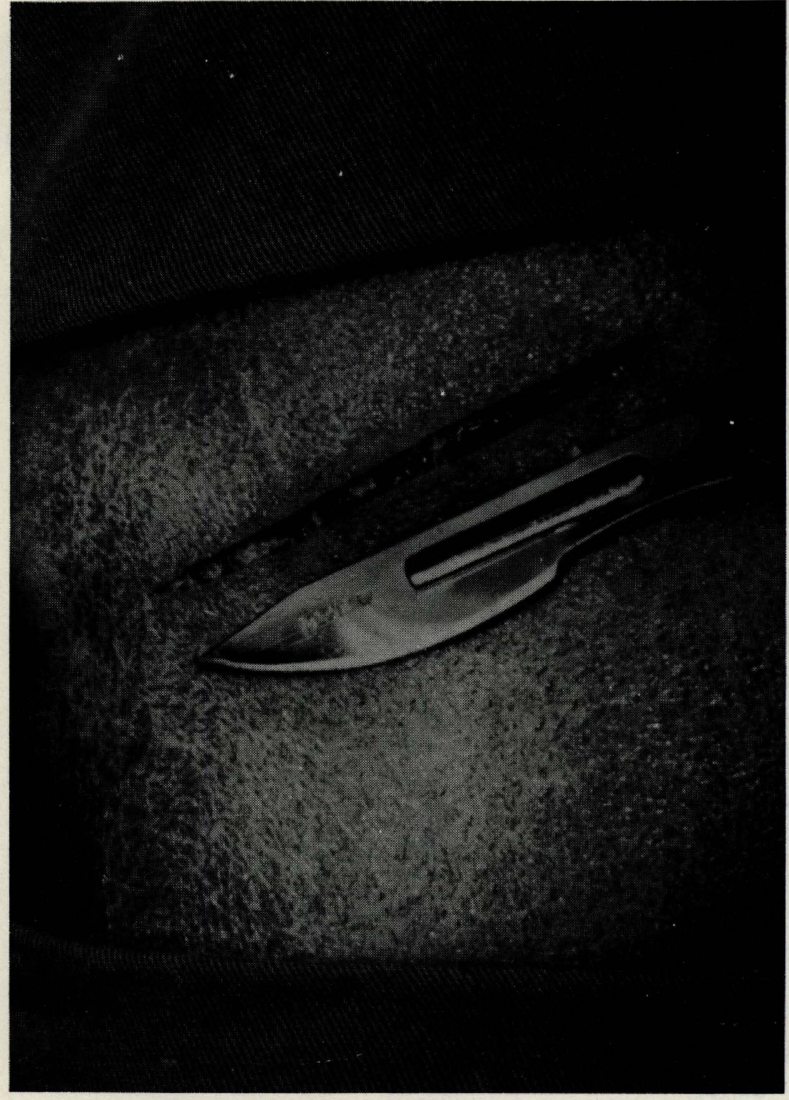

Fig. 3 - Incisão com bisturi na borda inferior da escápula.

intercostal, com o cuidado de não perfurar a pleura parietal. As costelas foram separadas por um auxiliar com um par de Farabeufs. Um afastador torácico, escolhido de acordo com o tamanho da criança, foi colocado afastando ainda mais as costelas (Figura 4). Com auxílio de bolinha de gaze montada em pinça Moynihan, a pleura parietal foi descolada do gradil costal, luxando o pulmão. Cuidado especial foi tomado ao dissecar a pleura sobre a aorta, ponto mais comum de ocorrência das perfuraçōes pleurais. A camada adventícia deve ser dissecada em direção à artéria pulmonar, levando em conjunto a pleura e o nervo vago. Já sobre o canal arterial, o nervo laríngeorecorrente foi visibilizado e afastado. A administração endovenosa de nitroprussiato de sódio permitiu a realização de hipotensão controlada, monitorada pela palpação da aorta ou por cateter na artéria radial. Dissecção cuidadosa deve ser feita para evitar intercorrências. Uma pinça Mixter média em ângulo reto é passada sob o canal, em direção à aorta. $O$ canal foi ligado por dois fios de Poliester $2 / 0$ passados individualmente (Figura 5). Uma sutura transfixante de Polipropileno $4 / 0$ foi feita e atada entre os dois fios de Mersilene (Figura 6). A palpação da aorta foi realizada certificando-se da ausência de frêmito. A administração de nitroprussiato de sódio foi desconti- 


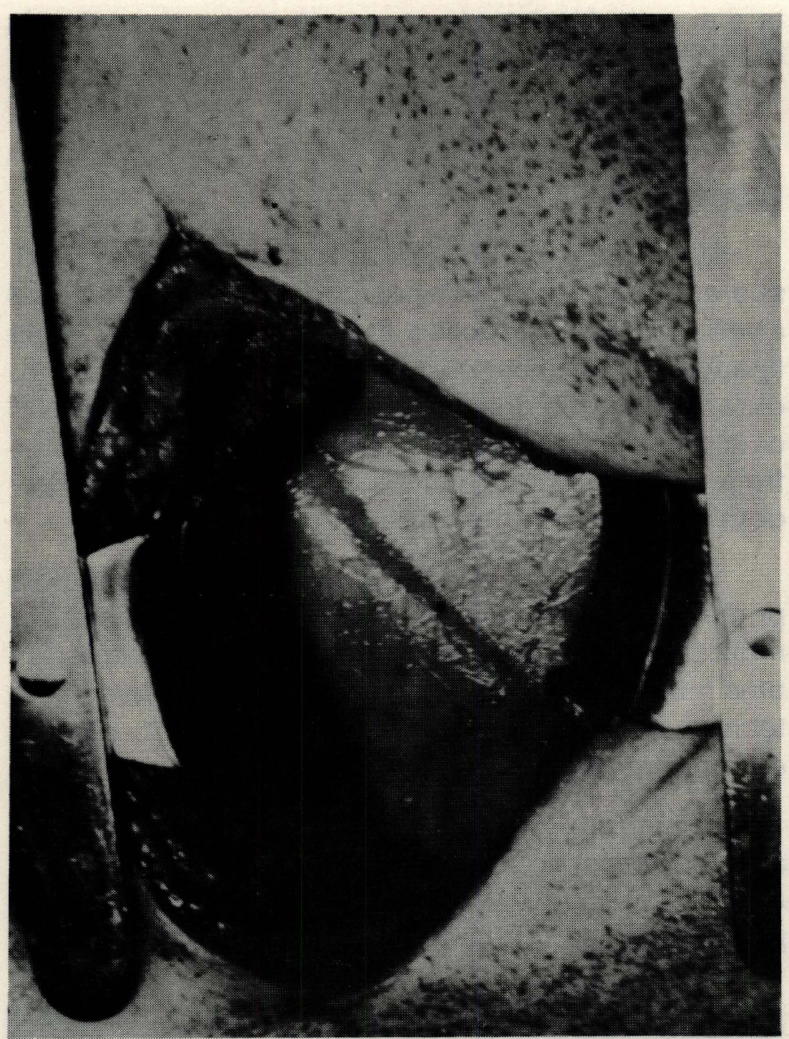

Fig. 4 - Colocação de afastador torácico de acordo com o tamanho da criança. Notar que a pleura parietal foi dissecada e se apresenta intacta.

nuada. O pulmão foi expandido, eliminando atelectasias causadas pelo afastamento. Não é necessária drenagem torácica. A aproximação das costelas foi conseguida com fios de Polyvicryl 0 . O fechamento foi realizado por planos com fios de Polyvicryl 3/0. Foi feito bloqueio intercostal com marcaína $0,5 \%$, sem adrenalina. Uma sutura intradérmica com fios de Polyvicryl $4 / 0$ concluiu o fechamento da incisão. Para curativo, usou-se micropore com aproximação das bordas (Figura 7 ).

As crianças submetidas à técnica permaneceram no hospital de dois a quatro dias após a alta da unidade de terapia intensiva. A permanência foi necessária para observação e complementação da antibioticoprofilaxia. No momento da alta hospitalar, a mãe foi instruída a fazer profilaxia para endocardite bacteriana por seis meses.

No pós-operatório tardio, os pacientes foram consultados pelo cardiologista pediátrico, para reavaliação, consistindo de interrogatório, exame físico, radiografia de tórax e ecodopplercardiograma.

\section{Recuperação Anestésica}

A criança foi colocada em decúbito dorsal, ces-

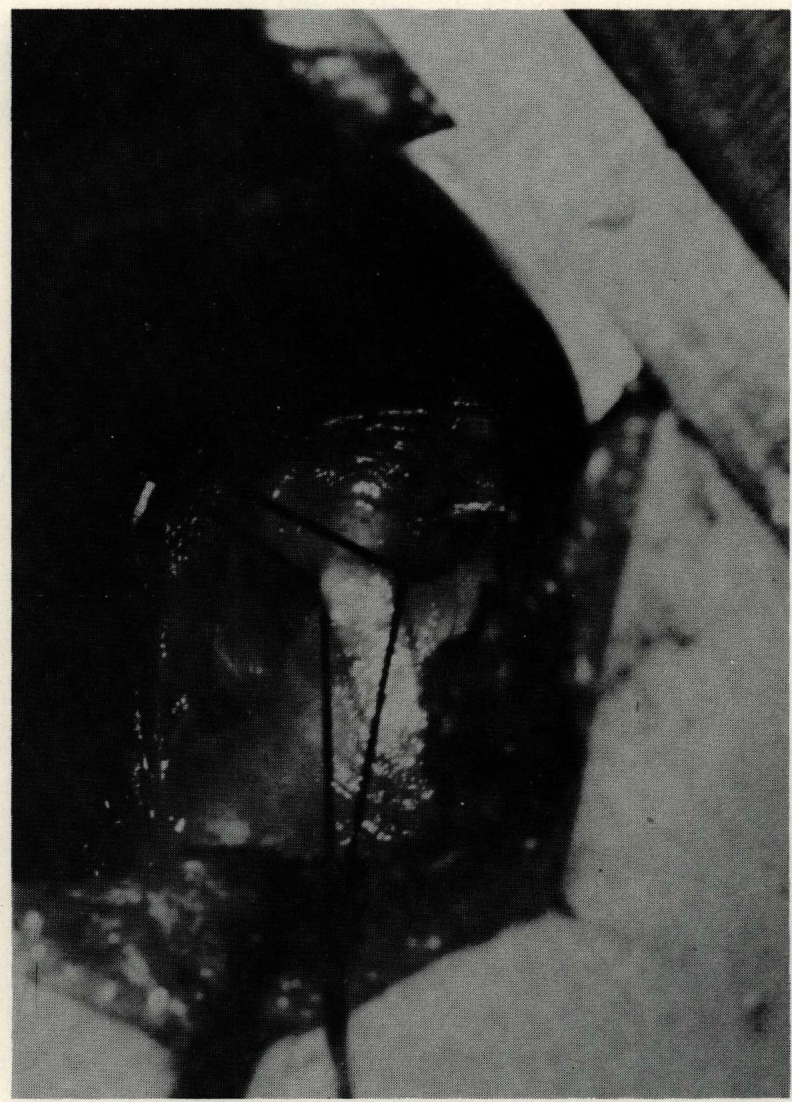

Fig. 5 - Fotografia intra-operatória mostrando canal arterial dissecado e laçado por dois fios de Poliester 2.0.

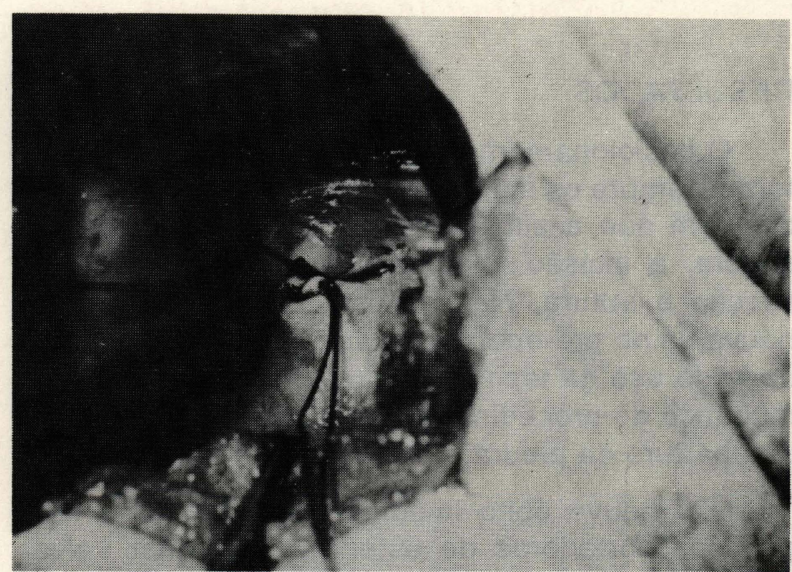

Fig. 6 - Fotografia intra-operatória mostrando canal arterial já ligado e com ponto transfixante de Polipropileno 4.0 amarrado.

sando-se a administração do anestésico inalatório no ar expirado. Uma vez em respiração espontânea, o paciente foi extubado e levado para a unidade de terapia intensiva, onde permaneceu em observação até a completa recuperação da anestesia, quando foi liberada para o quarto (duas ou três horas após), em companhia dos familiares. A dor nāo foi proeminente e o uso de anestésicos foi mínimo. 
SANTOS, J.L.V.; BRAILE, D.M.; ARDITO, R.V.; ZAIANTCHICK, M.; SOARES, M.J.F.; RADE, W.; LEAL, J.C.F.; JACOB, J.L.B.; HASSEN SOBRINHO, S. - Ligadura do canal arterial: técnica extrapleural. Rev. Bras. Cir. Cardiovasc., 7(1):14-21, 1992.

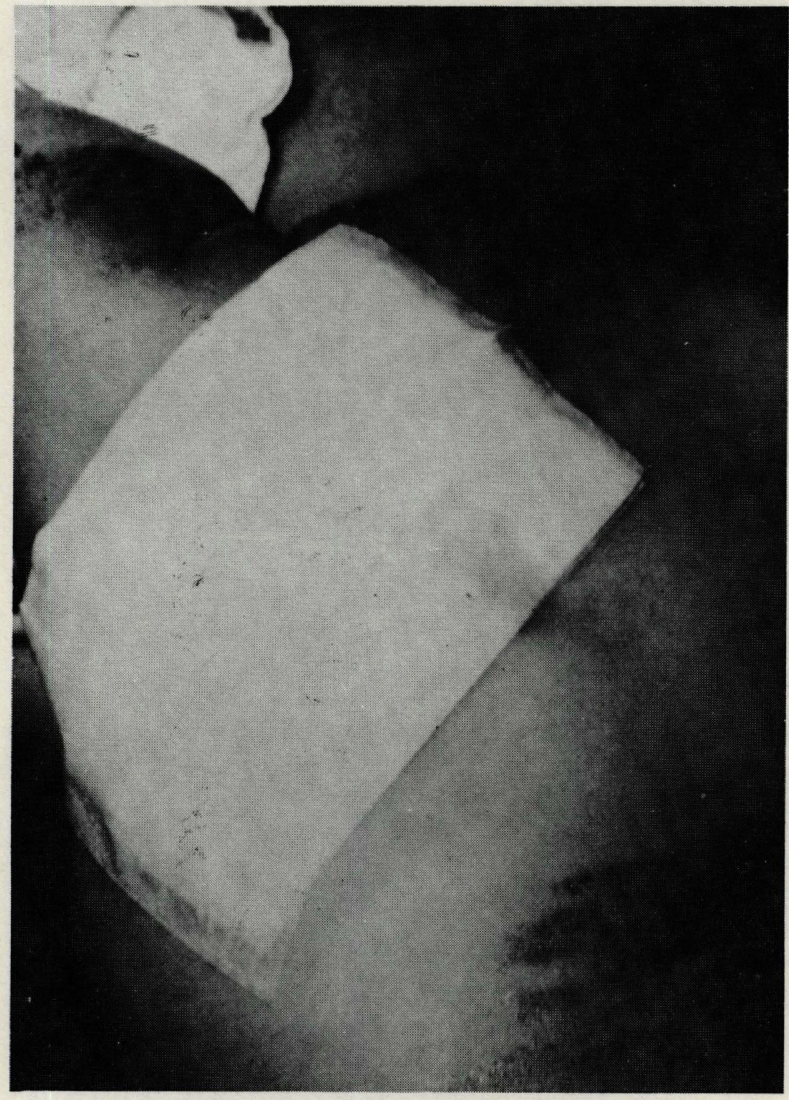

Fig. 7 - Fotografia pós-operatória mostrando pequeno curativo com micropore aproximando as bordas da incisão.

\section{RESULTADOS}

O posicionamento e a abordagem das crianças foram sempre os mesmos. Em canais arteriais grandes, em que a simples ligadura não se mostrava segura, a incisão foi ampliada para a técnica de secção e sutura. Nesses casos (sete pacientes), houve, já no pré-operatório, suspeita da impossibilidade do uso da técnica estrapleural. Mesmo com a mudança do procedimento, não houve necessidade de abertura da pleura e/ou drenagem torácica.

Não houve óbito imediato ou tardio. Das seis crianças portadoras de estenose pulmonar valvar associada, duas foram submetidas a dilatação por cateter-balāo 60 dias após a cirurgia. Todas as crianças portadoras de cardiopatia congênita associada permanecem em acompanhamento clínico.

O seguimento foi de $100 \%$ e variou de sete a 30 meses, com média de 18,5 meses. Todas as crianças permanecem em grau I da NYHA. Não houve infecção hospitalar ou comunitária no grupo.

A cicatriz cirúrgica obtida em todos os casos (Figura 8) mostra que o trauma e a discriminação tardia, que, fatalmente, ocorrem numa toracotomia ampla, passam a inexistir.

\section{DISCUSSĀO}

WILCOX \& PETERS ${ }^{21}$ apresentaram, em 1966, sua experiência de 14 anos com a cirurgia do canal arterial: $30 \%$ por secção e sutura e $70 \%$ por tripla ligadura. DAVIES et alii ${ }^{4}$ também optaram pela ligadura, na maioria dos casos. WRIGTH \& NEWMAN ${ }^{22}$ concordam com a ligadura, mas alertam para ductos frágeis, largos, calcificados e hipertensos, que podem se apresentar em diferentes idades.

Em 1978, TRAUGOTT et alii ${ }^{19}$ propuseram, pela primeira vez, a técnica de ligadura com clip metálico, nas crianças prematuras e naquelas que não toleram uma mínima retração pulmonar. Mais tarde, a mesma técnica foi referida por outros autores ${ }^{2,10,14}$, sempre em prematuros.

A ligadura com clips metálicos foi tentada, em alguns casos do grupo estudado, e abandonada. Acreditamos que esta seja uma boa opção para prematuros, conforme publicado por MEIER et alii ${ }^{14}$,

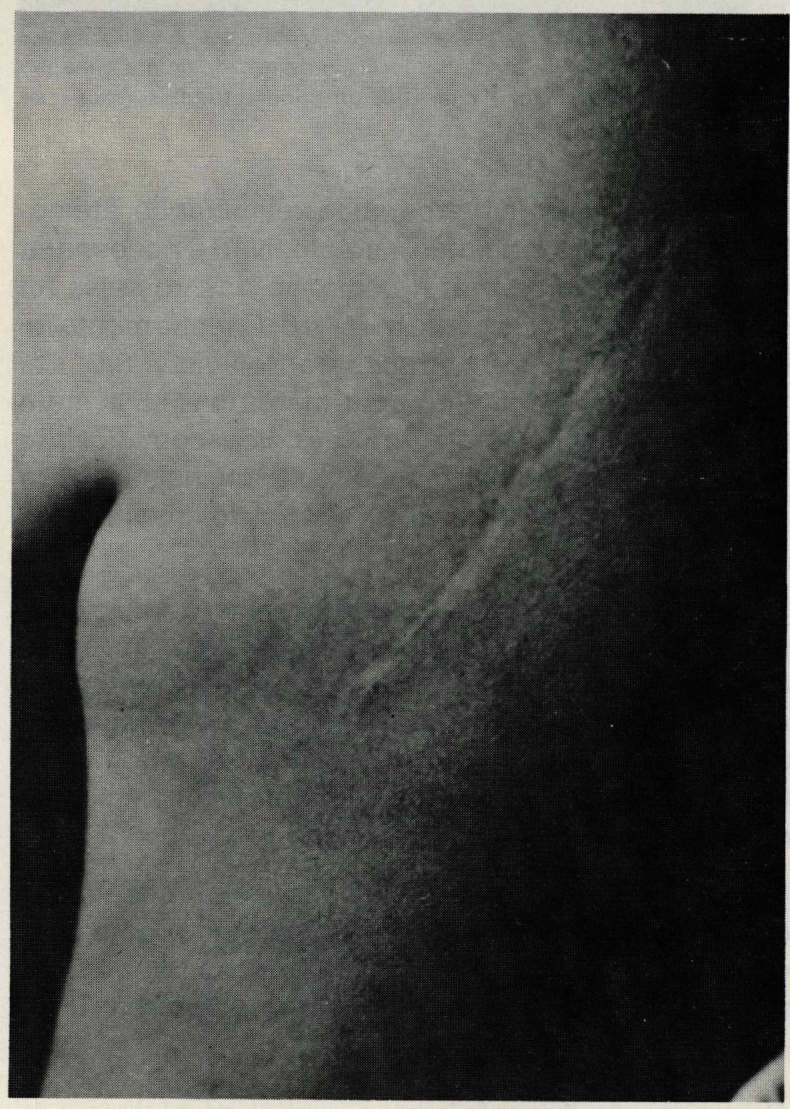

Fig. 8 - Fotografia mostrando cicatriz cirúrgica pós-operatória. 0 trauma e a discriminação tardios serão minimizados pelo aspecto cicatricial. 
SANTOS, J.L.V.; BRAILE, D.M.; ARDITO, R.V.; ZAIANTCHICK, M.; SOARES, M.J.F.; RADE, W.; LEAL, J.C.F.; JACOB, J.L.B.; HASSEN SOBRINHO, S. - Ligadura do canal arterial: técnica extrapleural. Rev. Bras. Cir. Cardiovasc., 7(1):14-21, 1992.

apesar da crítica feita por SALLUN ${ }^{16}$ pela estigmatização futura em radiografias a que as crianças possam ser submetidas. Em nossa experiência, os clips foram abandonados porque operamos crianças a termo e em bom estado clínico. Essas crianças suportaram, facilmente, a mobilização pulmonar, permitindo a passagem de pinça Mixter sob o canal e sua ligadura.

BRANDT et alii ${ }^{1}$, operando crianças prematuras, chamam a atenção para a ocorrência de displasia broncopulmonar no pós-operatório tardio. Da mesma forma, WAGNER et alii ${ }^{20}$, atuando também nessa faixa etária e seguindo tardiamente 268 crianças, concluem que a ligadura do canal arterial deve ser indicada por mudar a evolução natural da doença, com baixa mortalidade.

A insuficiência cardíaca congestiva e a síndrome respiratória aguda são relatadas por vários autores 3,6,13-15, que, utilizando as diversas técnicas cirúrgicas, mudaram o prognóstico de seus pacientes.

A paralisia de corda vocal foi relatada por DAVIS et alii ${ }^{5}$ e FAN et alii ${ }^{7}$, como uma grave complicação cirúrgica. Outra complicação, fluxo residual tardio pelo ductus, foi relatada por TEE ${ }^{18}$, revisando 625 casos operados na Malásia, com prevalência de $1,9 \%$.

Levando em conta o risco cirúrgico, as intercorrências pós-operatórias e a permanência intra-hospitalar, LATSON et alii ${ }^{11}$ publicaram, em 1989, a técnica de oclusão do canal arterial por cateterismo e dispositivos especiais no laboratório de hemodinâmica. A indicação da técnica foi feita pelos autores em $90 \%$ dos casos.

KHONSARI ${ }^{9}$ recomenda que a dissecção do canal deve ser cortante e não romba, por esta poder levar a lesão do canal e do nervo laríngeo-recorrente por distensão.

A pequena incisão a que as crianças foram submetidas pode ser perigosa, na opinião de muitos. A possibilidade de intercorrências, relatadas por SNAPRAGASAM et alii ${ }^{17}$, tais como sangramento, colapso pulmonar, quilotórax, rotura do canal etc, foi uma das preocupaçōes durante a realização e o desenvolvimento da técnica. A experiência adquirida mostrou que essas complicaçōes, relatadas na literatura, não ocorrem se alguns cuidados forem tomados no momento da ligadura, como: 1) dissecção paciente e cuidadosa da pleura, da aorta e do canal; 2) hipotensão controlada com nitroprussiato de sódio; 3) aperto da ligadura somente até o desaparecimento do frêmito; 4) ligadura transfixante com Polipropileno $4 / 0$ entre a dupla ligadura com Poliester $2 / 0$.
Não tivemos problemas quanto à anestesia, no grupo estudado. Todas as crianças foram induzidas com a mesma rotina, tendo bom comportamento intra-operatório. A recuperação anestésica teve início já durante a sutura do subcutâneo, com diminuição do anestésico inalatório. Todas foram extubadas na mesa cirúrgica e encaminhadas à unidade de terapia intensiva, para nebulização e observação.

No período estudado, apenas sete pacientes portadores de persistência do canal arterial não foram submetidas à técnica, sempre por contra-indicação pré-operatória: quatro por associação com coartação da aorta, um portador de canal atrioventricular comum total com hipertensão pulmonar, necessitando bandagem de artéria pulmonar, uma criança cujo canal tinha diâmetro maior que a aorta e um paciente com diagnóstico tardio aos 36 anos de idade.

A permanência hospitalar variou de dois a quatro dias após a alta da unidade de terapia intensiva. A curta permanência hospitalar é explicada pela morbidade nula representada por este procedimento. A ausência de drenagem torácica foi importante para esse resultado.

A história clínica, exame físico, radiografia de tórax e ecodopplercardiograma, realizados pelo cardiologista pediátrico por ocasião da avaliação clínica pós-operatória, foram ítens importantes para afastar complicações possíveis após a cirurgia: fluxo residual pelo canal, atelectasia pulmonar, lesão do nervo laríngeo recorrente, infecção etc. Nenhuma dessas complicaçōes foi encontrada no grupo operado.

A facilidade da técnica levou-nos a torná-la rotina para operar crianças pequenas e de baixo peso. Nestas, a cirurgia é mais simples e fácil: 1) por melhor afastamento do gradil costal: 2) por facilidade na dissecção pleural e do canal; 3) pela menor profundidade da caixa torácica.

\section{CONCLUSÕES}

A ligadura extrapleural do canal arterial é uma das muitas técnicas utilizadas atualmente para correção desta cardiopatia congênita. Em nossa experiência, mostrou-se benigna quanto ao intra e pós-operatório, não apresentando morbidfade e/ou mortalidade imediata ou tardia no grupo de crianças operadas durante um período de dois anos. Deve ser utilizada como técnica de escolha na correção da persistência do canal arterial, principalmente em crianças de baixa idade e peso. 
SANTOS, J.L.V.; BRAILE, D.M.; ARDITO, R.V.; ZAIANTCHICK, M.; SOARES, M.J.F.; RADE, W.; LEAL, J.C.F.; JACOB, J.L.B.; HASSEN SOBRINHO, S. - Ligadura do canal arterial: técnica extrapleural. Rev. Bras. Cir. Cardiovasc., 7(1):14-21, 1992.

\section{RBCCV 44205-157}

SANTOS, J.L.V.; BRAILE,D.M.; ARDITO,R.V.; ZAIANTCHICK,M.; SOARES,M.J.F.; RADE,W.; LEAL,J.C.F.; JABOR, J.L.B.; ASSEN SOBRINHO,S. - Ligation of patent ductus arteriosus: extrapleural technique. Rev. Bras. Cir. Cardiovasc., 7(1):14-21,1992.

ABSTRACT: The authors describe the surgery for patent ductus arteriosus (PDA) with triple ligature through the extrapleural path. After general anesthesia, the child has his position in the right lateral and a little ventral decubitus. A small incision is made in the inferior limit of the scapula. The pleura is dissected and the PDA exposed, double ligated with Polyester $2 / 0$ and transfixed and tightened with Polypropilene $4 / 0$. With this technique it is not necessary to have a chest tube. Fourty seven patients with PDA were submitted to surgery between September 1988 and August 1990; in 40 patients it was possible to do this type of surgery. Ages ranged from 4 months to 11 years, with mean age of $3.1 \pm 3.0$ years. Twenty children were less than one year old. There were 24 females and 16 males. No cases of intra or post-operative complications were registered. The follow-up ranged from 7 to 30 months (mean 15.3 months). All children were submitted to clinical evaluation, thoracic Rx and ecocopplercardiography. No patients presented recanalization or other complications. This experience showed that this tecnique is reliable, efective and it does not present immediate and late morbility or mortality. This technique can be safely used in younger as well as older children.

DESCRIPTORS: patent ductus arteriosus, surgery.

AGRADECIMENTO: Ao Dr. Randas José Vilela Batista, Curitiba, Paraná, por ter introduzido e estimulado o desenvolvimento desta técnica em nosso Serviço.

\section{REFERÊNCIAS BIBLIOGRÁFICAS}

1 BRANDT, B.; MARVIN, W.J.; EHRENHAFT, J.L.; HEINTZ, S.; DOTY, D.B. - Ligation of patent ductus arteriosus in premature infants. Ann. Thorac. Surg., 32:167$172,1981$.

COSTA, M.G.; VASCONCELOS, F.; SILVEIRA, C.A.; GASPAR, E.; GODOY, G.; GARRET, M.; PEREIRA, R.; MENEZES, A.; COSTA, L.G.; SELVA, A. - Correção cirúrgica da persistência do canal arterial utilizando "clip" cirúrgico: apresentação da técnica e relato de caso. Rev. Cir. Cardiovasc., 2:91-94, 1988.

COSTER, D.D.; GORTON, M.E.; GROOTERS, R.K.; THIEMAN, K.C.; SCHENEIDER, R.F.; SOLTANZADEH, $\mathrm{H}$. - Surgical closure of the patent ductus arterious in neonatal intensive care unit. Ann. Thorac. Surg., 48:386:389, 1989.

DAVIES, D.C.; ADEBO, O.A.; OSINOWO, O. - Surgical treatment of persistent ductus arterious at the University College Hospital, Ibadan. East Afr.Med.J., 17:340$342,1989$.

DAVIS, J.T.; BACIEWICZ, F.A.; SURIYAPA, S.; VAUTHY, P.; POLAMREDDY, R.; BARNETT, B. - Vocal cord paralysis in premature innfants indergoing ductal closure. Ann. Thorac. Surg, 46:214-215, 1988.
Persistencia del conducto arterioso: posibilidades diagnósticas y terapéuticas en nuestro medio. Rev. Cubana Ped., 57:179-191, 1985.

7 FAN, L.L.; CAMPBELL, D.N.; CLARKE, D.R.; WASHINGTON, R.L.; FIX, E.J.; WHITE, C.W. - Paralyzed left vocal cord associated with ligation of patent ductus arteriosus. J. Thorac. Cardiovasc. Surg., 98:611$613,1989$.

8 GROSS, R.E. \& HUBBARD, J.P. - Surgical ligation of a patent ductus arteriosus. JAMA, 112:729-731, 1939.

9 KHONSARI, S. - Atlas de cirurgia cardíaca: cuidados com a técnica operatória. São Paulo, Ed. Santos, 1990. p. 158-164.

10 KRON, I.L.; MENTZER, R.M.; RHEUBAN, K.S.; NOLAN, S.P. - A simple, rapid technique for operative closure of patent ductus arteriosus in the premature infant. Ann. Thorac. Surg., 37:422, 1984.

11 LATSON, L.A.; HOFSCHIRE, P.J.; HUGLER, J.D.; CHEATHAM, J.P.; GUMBINER, C.H.; DANFORD, A.A. - Transcatheter closure of patent ductus arteriosus in pediatric patients. J. Pediat., 115:549-553, 1989.

12 MACRUZ, R. \& SNITCOWSKY, R. - Cardiologia pediátrica. São Paulo, Sarvier, 1983. 782p.

13 MADIYONO, B.; OESMAN, I.N.; SATROASMORO, S.; PUTRA, S.L.; SOELAIMAN, E.J.; RACHMAD, K.B. Patent ductus arteriosus before and after surgery. Paedriatr. Indonesia, 29:39-51. 1989.

14 MEIER, M.A.; JAZBIK, W.; OLIVEIRA, J.A.; ASSUMPÇÃO, C.R.; FAGUNDES, M.L.A.; SILVA, J.C.; BARBOSA, R.C. - Cirurgia cardíaca em prematuros extremos e 
recém-nascidos de baixo peso. Rev. Bras. Cirur. Cardiovasc., 4:9-20, 1989.

15 REYES, R.M.A.; WEBWE, M.R.; VELA, J.E.; FLAMAND, E.L. - Resultados del tratamiento quirúrgico del conducto arterioso en el neonato com síndrome de dificultad respiratoria: estudio de 18 casos. ActaPediatric. Mex., 7: 48-55, 1986.

SALLUN, F.S. - Carta ao Editor. Rev. Cir. Cardiovasc., 2: $49,1989$.

17 SIVAPRAGASAM, S.; SPENCER, H.; BHOORASINGH, $P$. - Patent ductus arteriosus: results of operative intervention (1953-1984). West India Med. J., 34:180$183,1985$.

18 TEE, C.T.T. - Surgery for the persistent ductus arteriosus: a review of 625 cases. Med. J. Malaysia, 42:104$109,1987$.
19 TRAUGOTT, R.C.; WILL, R.J.; SCHUCHMANN, G.F.; TREASSURE, R.L. - A simplified method of ligation of patent ductus arteriosus in premature infants. Ann. Thorac. Surg., 29:263, 1980.

20 WAGNER, H.R.; ELLISON, C.; ZIERLER, S.; LANG, P.; PUROHIT, D.M.; BEHRENDT, D.; WALDHAUSEN, J.A. - Surgical closure of patent ductus arteriosus in 268 preterm infants. J. Thorac. Cardiovasc. Surg., 87:870-875, 1984.

21 WILCOX, B.R. \& PETERS, R.N. - The surgery of patent ductus arteriosus: a clinical report of 14 years' experience without an operative death. Ann. Thorac. Surg. 2:126131, 1967.

22 WRIGTH, J.S. \& NEWMAN, D.C. - Ligation of the patent ductus: technical considerations at different ages. J. Thorac. Cardiovasc. Surg. 75:695-698, 1978. 